\title{
Percepción del logro de las habilidades fonoaudiológicas y del requerimiento del mercado laboral al egreso de Fonoaudiólogos de la Universidad de Chile
}

Eduardo Fuentes L. Fonoaudiólogo

Programa de Doctorado en Salud Pública, Escuela de Salud Pública,

Universidad de Chile Facultad de Medicina, UDA Ciencias de la Salud, carrera de

Fonoaudiología, Pontificia Universidad Católica de Chile

\author{
Perception of the achievements of speech and \\ language therapists abilities and labour marker \\ demands in speech and language therapists \\ graduated from the Universidad de Chile
}

\section{RESUMEN}

En nuestro medio existe escasa evidencia acerca del nivel de formación alcanzado por parte de los fonoaudiólogos egresados de las universidades chilenas, así como también del requerimiento que dichos profesionales perciben desde el mercado laboral. Objetivo: Establecer la percepción que tienen los fonoaudiólogos de la Universidad de Chile respecto de la manera en que su formación es o no adecuada a los requerimientos laborales del medio. Método: Se aplicó un cuestionario a 60 fonoaudiólogos egresados de la Universidad de Chile que consultaba tanto sobre la percepción de desarrollo al egreso, como de la percepción en torno al requerimiento laboral. Los sujetos se dividieron en dos grupos de acuerdo con las modificaciones curriculares realizadas por esta universidad. Resultados: Al comparar ambas percepciones se obtuvo una diferencia significativa entre ellas. Dicha diferencia se mantuvo en seis habilidades fonoaudiológicas consideradas en esta investigación, sin embargo, al analizar cada una por separado, las diferencias varían entre sí y respecto del promedio global. Conclusiones: La percepción del requerimiento laboral es mayor que la percepción de la formación universitaria recibida. Además, la diferencia entre ambas percepciones se ve influenciada por el tipo de habilidad fonoaudiológica y también por el grupo al cual pertenecía el encuestado.

Palabras claves: relación empleo-formación fonoaudiológica, formación profesional, mercado laboral.

\begin{abstract}
In our field, there exists little evidence related to the level of formation achieved by the speech and language therapist graduates from Chilean universities, nor to the requirements which said professionals perceive from the labour market. Objective: To establish the perception which the Speech and Language Therapists have as to the extent that their formation meets the demands of the field. Method: Sixty Speech and Language Therapist graduates from the Universidad de Chile completed a questionnaire. The subjects were grouped into 2 sub-groups according to curricular modifications made by that university. Results: On comparing the perception of the achievement and the requirement on the part of the labour market, a clear difference can be seen in the results of the surveys. This difference is evident in all six speech and language therapy abilities considered when analysed not only with the difference between categories but also globally. Conclusions: The perception of the demands of the labour market is greater than the perception of the levels of formation received at university. Furthermore, the difference between both perceptions is influenced by the type of speech and language therapy ability and by the group to which it belongs.
\end{abstract}

Key words: work-formation relation, professional formation, labor market. 


\section{Introducción}

Hoy en día una gran cantidad de universidades que dictan la carrera de Fonoaudiología se encuentra rediseñando sus perfiles profesionales con el objetivo de adscribirse a un modelo de formación basado en competencias,. Se han descrito diversas fortalezas de este por sobre el modelo tradicional, dentro de las que se encuentra el hecho de permitir a la universidad dar fe de los aprendizajes logrados por parte de sus estudiantes y facilitar la incorporación temprana de los egresados al mercado laboral ${ }^{1}$. Así la construcción de un perfil profesional no solo busca proporcionar el sello propio de la casa de estudios a la formación ofrecida, sino también demostrar sus logros y desarrollos en el plano del conocimiento. En paralelo a dicho proceso de transformación curricular muchas de estas instituciones de educación superior investigan igualmente cuáles son las características de las prácticas profesionales actuales y las tendencias de la profesión $^{2}$. Es así como los empleadores son invitados a participar en la formulación y validación de las competencias profesionales planteadas en los nuevos perfiles profesionales. Al I incluirlos, las universidades esperan lograr una mayor sintonía no solo con el mercado laboral, sino que además aspiran a cumplir con las expectativas que la sociedad tiene respecto de los profesionales que forman.

El europeo es uno de los contextos más relevantes en donde se ha sistematizado la consulta a los egresados universitarios acerca su inserción laboral. Como ejemplo de ello es posible mencionar la aplicación a finales de los 90 de la encuesta
CHEERS (Careers after Higher Education - A European Research Survey) en una serie de países de ese continente y en la que se consultó a más de 36.000 graduados de 11 naciones europeas y Japón. Al respecto, la encuesta solicitó información acerca de las demandas y de la formación entregada sobre la base de 32 competencias $^{3}$. En este caso, los graduados europeos debían valorar (en una escala ordinal de 1 a 5) las competencias que eran requeridas en sus puestos de trabajo, así como también el nivel de logro de dichas competencias ${ }^{3}$. Los resultados mostraron que los puestos laborales exigían un conjunto de competencias entre las que podemos destacar aquellas relacionadas con la capacidad de negociación, planificación, coordinación y organización y con asumir responsabilidades, las que no eran desarrolladas por las instituciones formadoras ${ }^{4}$.

En el caso de Chile, según Brunner y Uribe $\left(2007^{5}\right)$ las instituciones formadoras «no tienen por costumbre informar acerca de lo que ocurre con sus alumnos entre el momento en el que se matriculan y en el que se gradúan. Tampoco existe una adecuada información acerca de los resultados del aprendizaje (el capital humano realmente adquirido), ni sobre el desempeño de los graduados al incorporarse al mercado laboral».

La situación en la carrera de Fonoaudiología no difiere de lo expresado anteriormente, ya que aún no se han realizado investigaciones respecto de la percepción de logro de los estudiantes al momento de egresar, de las habilidades y destrezas expresadas en los perfiles profesionales, así como tampoco de las exigencias que experimentan los egresados al 
incorporarse al mercado laboral. A lo anterior se suma el hecho de que actualmente solo la Universidad de Chile posee egresados con más de 15 años de experiencia, lo que dificulta la investigación en este ámbito.

\section{Formación universitaria y demandas del mercado laboral}

Como se mencionó anteriormente, en el contexto latinoamericano son escasos los reportes acerca de la inserción laboral de los profesionales universitarios. En Europa, en cambio, existen ejemplos de la aplicación de encuestas a gran escala para indagar acerca de este fenómeno, como es el caso de la mencionada encuesta CHEERS. En este caso, en la publicación realizada por Mora (2003) y sobre la base de los resultados que arrojó dicha encuesta, Ilama la atención el hecho de que los graduados universitarios valoraran mayormente las competencias sociales (trabajar en equipo, adecuación al trabajo, apreciar otros puntos de vista, etc.) por sobre las competencias «más duras» o disciplinarias y que los principales déficits respecto de las demandas laborales se encontraran en las competencias participativas (capacidad para liderar, tomar decisiones y asumir responsabilidades, entre otras). Las especializadas (fundamentalmente los conocimientos) representan el conjunto de competencias menos valorado, tanto personalmente como en el puesto de trabajo $^{6}$. Cabe mencionar que en este continente la crítica más común por parte de los empleadores hacia los graduados universitarios es la falta de conocimientos prácticos y especializados ${ }^{7}$.
Otros resultados obtenidos utilizando

metodologías cualitativas complementan lo anteriormente mencionado. En efecto, un estudio realizado por la Agencia Nacional de Evaluación de la Calidad y Acreditación de España, ANECA (2009), respecto de los procesos de inserción laboral de los titulados universitarios españoles evidenció la dificultad que estos enfrentan en este ámbito y también la brecha existente entre la universidad y el mundo del trabajo. Por ejemplo, los graduados manifestaron su creencia en la irrelevancia del expediente académico para la búsqueda de empleo y consideraron, además, que todo lo necesario para el trabajo podía ser adquirido dentro del propio desempeño laboral, catalogando la formación universitaria en términos generales como excesivamente generalista y teórica ${ }^{8}$. Alonso, Rodríguez y Nyssen $\left(2008^{9}\right)$, utilizando también un paradigma cualitativo, observaron que los jóvenes egresados españoles describían la formación universitaria como: «excesivamente teórica y ensimismada y no conectada adecuadamente con un conocimiento útil capaz de responder a las necesidades de la sociedad en general y del mercado laboral en particular».

\section{Enfoque tradicional de formación}

Durante el último siglo la educación se ha basado en un modelo estándar denominado por algunos autores como del tipo «Cohorte». Para Bell y Mitchell $\left(2000^{10}\right)$ dicho término alude al hecho de que en este modelo los cursos están constituidos por un número relativamente grande de estudiantes que se desplazan, como grupo y al mismo ritmo, a través del plan de estudios. Algunas de las ventajas de este 
modelo incluyen la facilidad para organizar el tiempo, ya que todos los estudiantes de determinada cohorte se encuentran en el mismo lugar, al mismo tiempo. También facilita el uso eficiente del tiempo por parte del profesor, ya que agrupa las necesidades de aprendizaje de un número significativo de estudiantes. Sin embargo, también existen desventajas, dado que al estar diseñado para trabajar con grupos de estudiantes se pueden descuidar las necesidades individuales de aprendizaje. Esto puede ser un problema en la formación profesional, donde los alumnos presentan distintas experiencias y habilidades para el aprendizaje.

Según Peluffo $\left(2005^{11}\right)$, el modelo tradicional se caracteriza por un diseño curricular organizado en torno a asignaturas que apuntan a aprendizajes formales en una disciplina del conocimiento. Dicho perfil es diseñado desde las capacidades y características que la academia le asigna a cada egresado de sus carreras o programas. Los programas de estudios están centrados en las actividades docentes y en el logro de objetivos de formación para el desarrollo de capacidades principalmente académicas. En este modelo el rol del docente posee características tradicionales o frontales debido a que utiliza modelos pedagógicos centrados en la docencia y en lo académico; por el contrario, el alumno es considerado pasivo, ya que es dependiente del profesor y de su planificación.

Para Mora $\left(2004^{4}\right)$ el modelo tradicional es dominante en Latinoamérica, porque está diseñado para dar respuesta a las necesidades de un mercado laboral caracterizado por profesionales con escasa comunicación interdisciplinaria, por lo cual estos requieren competencias siempre específicas. Además, en este modelo las profesiones son estables, con exigencias que se mantienen a lo largo de la vida laboral. Debido a lo anterior, las universidades se limitan a enseñar el «estado del arte» en cada profesión.

\section{Proceso de innovación curricular en la Universidad de Chile}

Desde que en enero de 1978 se titulara la primera promoción de fonoaudiólogos de la Universidad de Chile se han realizado constantes reformas curriculares. Uno de los hitos más sobresalientes del proceso de innovación curricular ha sido el introducir el grado de licenciado en el año $1995^{12}$. Es así como se agregó un quinto año a la carrera, lo que trajo consigo un aumento considerable en la carga académica de los estudiantes.

Con el objetivo de implementar un nuevo currículo basado en competencias, la Escuela de Fonoaudiología de la Universidad de Chile conformó la Comisión de Innovación Curricular. Dicha comisión ha desarrollado tareas relacionadas con el levantamiento de las demandas formativas y donde las principales acciones fueron la investigación de diversas fuentes acerca de dichas demandas, la construcción de las matrices de análisis, la determinación de necesidades y los compromisos formativos. Además, se elaboró una proposición declarativa del perfil de egreso, para lo cual se llevaron a cabo acciones tales como la determinación de dominios de formación y la declaración de competencias y subcompetencias. Finalmente, la 
Comisión ejecutó las tareas tendientes a organizar y estructurar el currículo ${ }^{13}$.

\section{Hipótesis, objetivos, variables}

En cuanto a la hipótesis de trabajo, el presente estudio plantea la existencia de diferencias entre la percepción que poseen los fonoaudiólogos acerca del dominio alcanzado al momento de egresar y el nivel de requerimiento experimentado durante el ejercicio laboral de las habilidades fonoaudiológicas. Dichas diferencias podrían ser explicadas por el modelo formativo de carácter tradicional utilizado por la institución formadora.

Por su parte, el objetivo de la presente investigación es establecer la percepción que tienen los fonoaudiólogos de la Universidad de Chile respecto de la manera en que su formación es o no adecuada a los requerimientos del medio. Lo anterior permitiría contrastar los resultados logrados por medio del enfoque educativo tradicional con las futuras modificaciones curriculares que la institución formadora pretenda implementar.

Por último, en cuanto a las variables, se entenderá por "percepción de logro al egreso» la valoración subjetiva que posee el profesional acerca del desarrollo de las habilidades fonoaudiológicas alcanzado en la formación universitaria; mientras que la «percepción de exigencia» se entenderá como la valoración subjetiva que posee el profesional respecto del requerimiento de las habilidades fonoaudiológicas por parte del mercado laboral.

\section{Método}

\section{Muestra}

La muestra estuvo conformada por 60 fonoaudiólogos egresados de la Universidad de Chile y que fueron educados dentro de un modelo tradicional de formación. La selección de los sujetos fue por conveniencia, del tipo no probabilística. De este modo, se invitó a participar en el estudio a fonoaudiólogos que asistieron a cursos y congresos llevados a cabo en el año 2009 y comienzos del 2010. Otro porcentaje de la muestra se obtuvo a través de consulta vía correo electrónico. Todos los encuestados realizaban su ejercicio profesional en diversas ciudades a lo largo de Chile.

En cuanto a las áreas de desempeño, el $42 \%$ de los participantes del estudio se ha desempeñado en solo una de las áreas propias de la carrera de Fonoaudiología. Por su parte, el $88 \%$ de los encuestados trabaja (o lo hizo a lo largo de su carrera profesional) en el área de lenguaje y habla en niños; el $45 \%$ en el área de voz; $37 \%$ en audición y $23 \%$ en el área de lenguaje y habla en adultos.

En relación con los sectores en donde los encuestados han ejercido profesionalmente, el $78 \%$ de ellos lo ha hecho en forma particular-privada; $70 \%$ en el sector de salud pública; $62 \%$ en docencia universitaria; $60 \%$ en una escuela de lenguaje; $42 \%$ en una escuela especial y el $20 \%$ en proyecto de integración.

Dentro de los antecedentes académicos relatados por los encuestados destaca que el $65 \%$ de ellos posee el grado de licenciado; mientras que el 
$37 \%$ posee el grado académico de magíster, o bien, lo está cursando, porcentaje que se repite para el caso de los encuestados que han cursado algún diplomado.

Otra característica importante considerada para el estudio fueron los años de experiencia laboral que poseían los profesionales. Es así como el rango varió entre uno y 33 años de ejercicio, mientras que el promedio fue de 15,5 años y la mediana alcanzó los 13,5 años, con una desviación estándar de 10,5 años.

Con el objetivo de observar los cambios en la formación que la carrera experimentó a lo largo de su desarrollo, se dividió la muestra en dos grupos (30 sujetos en cada uno). Dicha separación obedece a que en el año 1995 la Escuela de Fonoaudiología de la Universidad de Chile modificó su estructura curricular al introducir el grado de licenciado. Es así como el grupo №1 está conformado por fonoaudiólogos que egresaron posteriormente a la introducción del grado de licenciado y el grupo №2 está constituido por los que egresaron antes de dicha modificación curricular (lo cual implica que no obtenían grado académico al egresar).

\section{Instrumento}

El estudio consideró el uso de un cuestionario, el cual forma parte de la tesis para optar al grado de Magíster en Pedagogía Universitaria, desarrollada por los fonoaudiólogos Cáceres, Didier y Fuentes ${ }^{13}$ y que se describe más en detalle en las siguientes páginas.

\section{Descripción general del instrumento}

El cuestionario está constituido por dos secciones. La primera de ellas fue construida con el objetivo de caracterizar la muestra y constó de tres ítems: antecedentes personales (nombre, RUT, teléfono, nacionalidad y correo electrónico), antecedentes laborales (años de experiencia laboral y áreas de desempeño) y antecedentes académicos (títulos, cursos, especializaciones y grado académico). La segunda sección del cuestionario correspondió a una tabla de doble entrada, en donde en una de las columnas o entradas se debía valorar el logro de las habilidades al momento de egresar de la universidad $y$, en la otra, las exigencias laborales para cada una de las habilidades consultadas. Cada valoración se debía realizar en una escala de 1 a 5 , en donde el 1 correspondía a "bajo desarrollo o requerimiento» y el 5 a «alto grado de desarrollo o requerimiento» (ver Anexo).

\section{Modificaciones a la versión original del instrumento}

La versión original del cuestionario incluyó trece habilidades fonoaudiológicas, las que se obtuvieron al revisar los diferentes perfiles profesionales publicados por las universidades chilenas que imparten la carrera de Fonoaudiología. La validación del contenido del instrumento se realizó mediante una consulta a un grupo de cinco expertos en el área que respondieron a la solicitud de evaluar la pertinencia de los indicadores seleccionados, así como también la forma en que estos serían medidos. 
Luego de ello, se procedió a ajustar la primera versión del cuestionario y se aplicó finalmente en forma piloto a sujetos con características similares a los incluidos en la muestra ${ }^{13}$.

Con el objetivo de aportar en la validación de constructo del instrumento, la versión original del cuestionario fue sometida a un análisis factorial exploratorio, el que tuvo por finalidad encontrar las variables latentes que se presume constituyen la base de las variables observadas empíricamente ${ }^{14}$.

En un primer momento se obtuvieron las correlaciones entre la percepción de logro y la percepción de exigencia de las 13 habilidades fonoaudiológicas incluidas en la versión original del cuestionario. Considerando las características del instrumento se obtuvo la matriz de correlación a través de correlaciones policóricas. Luego se decidió eliminar aquellas habilidades con correlaciones bajas (coeficiente de correlación menor a 0.3) y altas (coeficiente de correlación mayor a 0.9), dado su bajo poder discriminante.

Posteriormente se extrajeron los factores que subyacen a las variables y las respectivas cargas de cada una de las preguntas (habilidades) del cuestionario. Lo anterior permitió detectar aquellas habilidades con cargas factoriales cruzadas, es decir, que poseían representación en más de un factor, y por ello, fueron eliminadas. Cabe destacar el hecho de que las habilidades fonoaudiológicas relacionadas con el proceso de investigación mostraron una pobre carga factorial y una elevada representación en más de un factor.
La solución factorial que no tuvo cargas cruzadas resultó estar compuesta por un total de 6 habilidades. El análisis exploratorio practicado a dichas habilidades evidenció dos factores que explicaban el $80 \%$ de la varianza. El primer factor dio cuenta del $41 \%$ de la varianza explicada y en él se agrupó la percepción de logro de las habilidades al momento de egresar, en tanto que el segundo factor dio cuenta del 39\% de la varianza explicada y en él se agrupó la percepción de requerimiento por parte del mercado laboral.

Con el objetivo de corroborar la validez de la solución factorial planteada se obtuvieron medidas acerca de la bondad de ajuste. En una primera instancia se determinó que esta no fue completamente adecuada $\left(X^{2}(23)=82.563, p<0.001\right)$. Sin embargo, el índice de ajuste CFI fue mayor al valor de 0.95 propuesto por $\mathrm{Hu}$ y Bentler ${ }^{15}$, alcanzando un valor de 0.98 y el índice de ajuste Tucker-Lewis (TLI) también fue elevado: 0.97. Además, la razón $\mathrm{X}^{2} / \mathrm{df}$ fue menor a 5 (3.58). Lo anterior podría estar indicando que los constructos «Percepción que poseen los fonoaudiólogos acerca del dominio alcanzado al egresar» y «Percepción del requerimiento experimentado en el ejercicio laboral de las habilidades fonoaudiológicas» son multifactoriales. En suma, las siguientes son las habilidades que finalmente fueron consideradas para evaluar tanto la percepción de logro como la percepción de requerimiento laboral: 
1. Evaluar el desarrollo comunicativo en las áreas de Voz, Audición, Habla y Lenguaje.

2. Establecer diagnósticos fonoaudiológicos en las áreas de Voz, Audición, Habla y Lenguaje.

3. Planificar la intervención terapéutica de acuerdo con las necesidades comunicativas de la persona y su entorno.

4. Implementar planes de intervención terapéutica de acuerdo con las necesidades comunicativas de la persona y su entorno.

5. Evaluar el plan de intervención terapéutica de acuerdo con las necesidades comunicativas de la persona y su entorno, readecuándolo cuando sea pertinente.

6. Desarrollar proyectos desde el ámbito fonoaudiológico tanto a nivel público como privado.

\section{Confiabilidad}

Con el objetivo de determinar la consistencia interna de cada uno de los ítems del instrumento, se calculó el coeficiente de confiabilidad alfa de Cronbach. El total de preguntas del cuestionario obtuvo un $\alpha=0.81$, lo que indicaría una buena consistencia interna del test. En cuanto a las preguntas que incluyó el primer factor, estas obtuvieron un $\alpha=0.89$, mientras que las que se agruparon en torno al segundo factor alcanzaron un $\alpha=0.71$.

\section{Pruebas estadísticas utilizadas}

Las variables cualitativas ordinales se describieron en cuanto a su frecuencia absoluta y porcentaje con respecto al total. En el caso de las variables continuas, cuando presentaron una distribución no normal se utilizó la mediana como estadígrafo descriptivo. Se reportó el promedio para las que presentaban una distribución normal. La distribución de la variable se constató mediante el Test de Shapiro-Wilk.
Al momento de realizar las comparaciones entre las variables, si ellas no poseían una distribución normal se utilizaron pruebas no paramétricas como el Test de rangos con signos por parejas de Wilcoxon. Por el contrario, si presentaban una distribución normal y se requería comparar diversos grupos (o habilidades) se utilizaron pruebas del tipo paramétrico como un ANOVA. Mediante esta última prueba se crearon modelos estadísticos ajustados según los predictores categóricos considerados (tipo de habilidad fonoaudiológica y grupo al cual pertenecía el encuestado). Finalmente, se realizaron los contrastes entre los distintos niveles de los predictores categóricos.

Por último, se utilizó un alfa de 0.05 para determinar la significancia en las pruebas estadísticas utilizadas. Se empleó el software STATA versión 12.1 para ejecutar las pruebas anteriormente mencionadas. 


\section{Resultados}

\section{Rendimiento de las habilidades fonoaudiológicas}

Los resultados en cada una de las habilidades fonoaudiológicas evidenciaron las diferencias existentes entre la percepción de logro al egreso y la percepción de requerimiento laboral por parte de los encuestados. En términos generales, para todas las habilidades consideradas fue evidente la mayor percepción de requerimiento por parte del mercado laboral, denotado por la ausencia de respuestas con valor 1 y 2 en el caso de dicha percepción, como se aprecia en la Tabla 1.

Tabla 1. Frecuencia absoluta y relativa de ambas percepciones

Habilidad Fonoaudiológica №1

\begin{tabular}{|c|c|c|c|c|c|}
\hline & Valor 1 & Valor 2 & Valor3 & Valor 4 & Valor 5 \\
\hline Desarrollo egreso & $1(1,7 \%)$ & $8(13,3 \%)$ & $13(21,7)$ & $23(38,3)$ & $15(25 \%)$ \\
\hline Requerimiento mercado & $0(0 \%)$ & $0(0 \%)$ & $3(5 \%)$ & $12(20 \%)$ & 45 (75\%) \\
\hline \multicolumn{6}{|c|}{ Habilidad Fonoaudiológica №2 } \\
\hline & Valor 1 & Valor 2 & Valor3 & Valor 4 & Valor 5 \\
\hline Desarrollo egreso & $1(1,7 \%)$ & $6(10 \%)$ & $11(18,3)$ & $27(45 \%)$ & $15(25 \%)$ \\
\hline Requerimiento mercado & $0(0 \%)$ & $0(0 \%)$ & $1(1,7 \%)$ & $9(15 \%)$ & $50(83,3 \%)$ \\
\hline \multicolumn{6}{|c|}{ Habilidad Fonoaudiológica №3 } \\
\hline & Valor 1 & Valor 2 & Valor3 & Valor 4 & Valor 5 \\
\hline Desarrollo egreso & $4(6,8)$ & $7(11,8 \%)$ & $17(28,3 \%)$ & $21(35 \%)$ & $11(18,3 \%)$ \\
\hline Requerimiento mercado & $0(0 \%)$ & $0(0 \%)$ & $1(1,7 \%)$ & $11(18,3 \%)$ & $48(80 \%)$ \\
\hline \multicolumn{6}{|c|}{ Habilidad Fonoaudiológica №4 } \\
\hline & Valor 1 & Valor 2 & Valor3 & Valor 4 & Valor 5 \\
\hline Desarrollo egreso & $2(3,3 \%)$ & $14(23,3 \%)$ & $14(23,3 \%)$ & $20(33,3 \%)$ & $10(16,7 \%)$ \\
\hline Requerimiento mercado & $0(0 \%)$ & $0(0 \%)$ & $2(3,3 \%)$ & $11(18,3 \%)$ & $47(78,3 \%)$ \\
\hline \multicolumn{6}{|c|}{ Habilidad Fonoaudiológica №5 } \\
\hline & Valor 1 & Valor 2 & Valor 3 & Valor 4 & Valor 5 \\
\hline Desarrollo egreso & $4(6,7 \%)$ & $15(25 \%)$ & $16(26,7 \%)$ & $15(25 \%)$ & $10(16,7 \%)$ \\
\hline Requerimiento mercado & $0(0 \%)$ & $0(0 \%)$ & $4(6,7 \%)$ & $10(16,7 \%)$ & $46(76,7 \%)$ \\
\hline \multicolumn{6}{|c|}{ Habilidad Fonoaudiológica №6 } \\
\hline & Valor 1 & Valor 2 & Valor 3 & Valor 4 & Valor 5 \\
\hline Desarrollo egreso & $22(36,7 \%)$ & $14(23,3 \%)$ & $17(28,3 \%)$ & $6(10 \%)$ & $1(1,7 \%)$ \\
\hline Requerimiento mercado & $2(3,3 \%)$ & $2(3,3 \%)$ & $13(21,7)$ & $20(33,3 \%)$ & $23(38,3 \%)$ \\
\hline
\end{tabular}

* El encuestado debía valorar cada percepción en una escala de 1 a 5.

Valor 1 indica bajo desarrollo al egreso o bajo requerimiento del mercado (según corresponda).

Valor 5 indica alto grado de desarrollo al egreso o alto requerimiento del mercado (según corresponda). 
Posteriormente se buscó establecer si existían diferencias en las percepciones de desarrollo al egreso con respecto a las del requerimiento laboral. En un primer momento y con el fin de facilitar el análisis de los datos, se realizó la sumatoria de las valoraciones para cada una de las habilidades fonoaudiológicas. Posteriormente, se constató que la sumatoria de la percepción de requerimiento por parte del mercado laboral se distribuía en forma no normal $(z=5.16, p<0.01)$ y normal, en el caso de la sumatoria de la percepción de desarrollo al egresar $(z=-0.63, p=0.73)$. Considerando lo anterior, se aplicó el Test de Wilcoxon, y se evidenció que existen diferencias estadísticamente significativas entre los puntajes der ambas percepciones $(z=6.38, p<0.001)$, siendo la percepción de requerimiento laboral mayor (Mediana $=29$ ) que la percepción de desarrollo al egresar de la universidad (Mediana $=21$ ), como se muestra en el Gráfico 1.

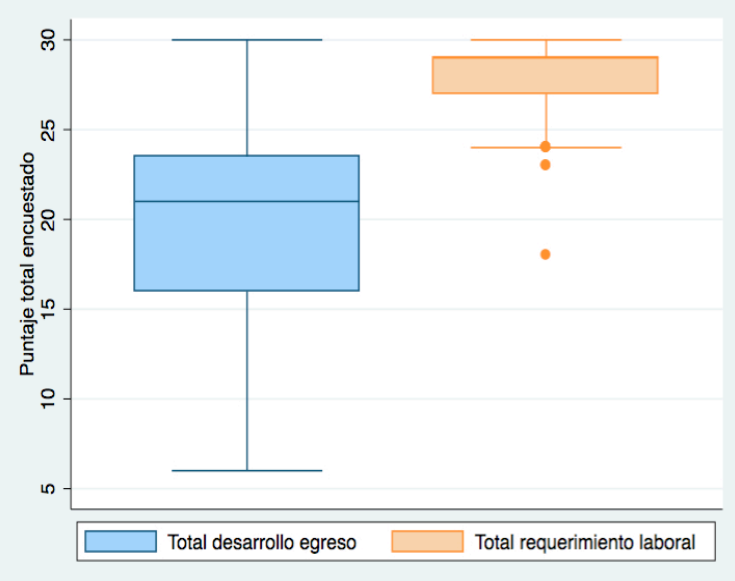

Gráfico 1. Comparación del puntaje total de ambas percepciones
Considerando la separación en dos grupos (efectuada según el egreso antes o después de la modificación curricular), se compararon los puntajes de cada una de las percepciones en cada grupo. En el grupo №1 (egresados con grado de licenciado), hubo diferencias estadísticamente significativas entre los puntajes de ambas percepciones $(z=3.01$, $\mathrm{p}<0.01)$; siendo la percepción de requerimiento laboral mayor (Mediana $=28,5$ ) que la percepción de desarrollo al egresar de la universidad (Mediana = 21). La diferencia también resultó significativa ( $z=$ $3.24, p<0.01$ ) en el grupo №2 (egresados sin grado de licenciado), donde la percepción de requerimiento laboral (Mediana $=29$ ) fue mayor que la percepción de desarrollo al egresar de la universidad (Mediana = 19,5), tal como se aprecia en el Gráfico 2.

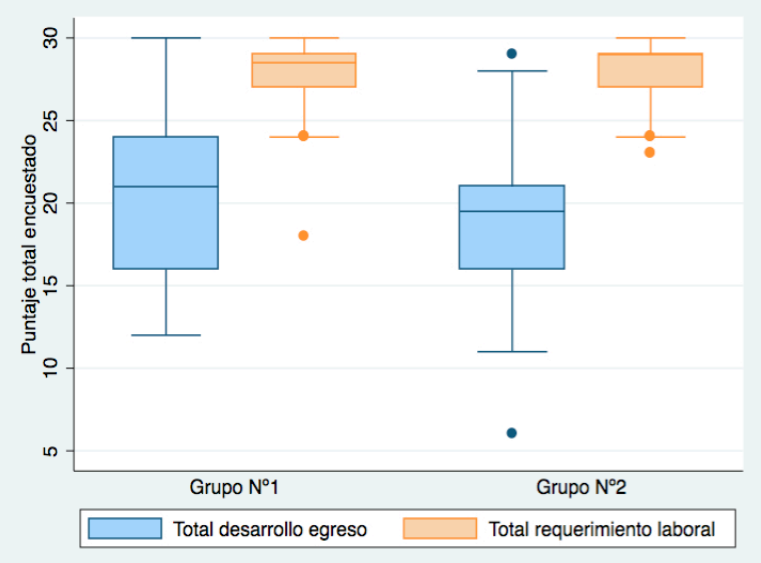

Gráfico 2. Comparación del puntaje total de ambas percepciones en los dos grupos. 


\section{Comparación de las diferencias entre ambas percepciones}

Interesaba también establecer en cuál de las seis habilidades consultadas existían mayores diferencias entre la percepción de requerimiento por parte del mercado y la percepción de desarrollo al egresar de la universidad. Para ello, en primer término, se obtuvo la diferencia aritmética entre ambas y se constató que dicha diferencia presenta una distribución normal ( $z=-1.86, p=0.969)$. Luego se aplicó un ANOVA para establecer si en cada una de las seis habilidades existían variaciones significativas en los promedios de las diferencias entre ambas percepciones. Los resultados evidenciaron que existen diferencias significativas en dos de las habilidades estudiadas $(F=4.41, p<0.001)$. Al utilizar el test de Scheffé, se constató que las diferencias significativas se encuentran entre las habilidades $1 \mathrm{y}$ $6(p<0.01)$, y entre esta última y la $2(p<0.05)$, como se advierte en el Gráfico 3.

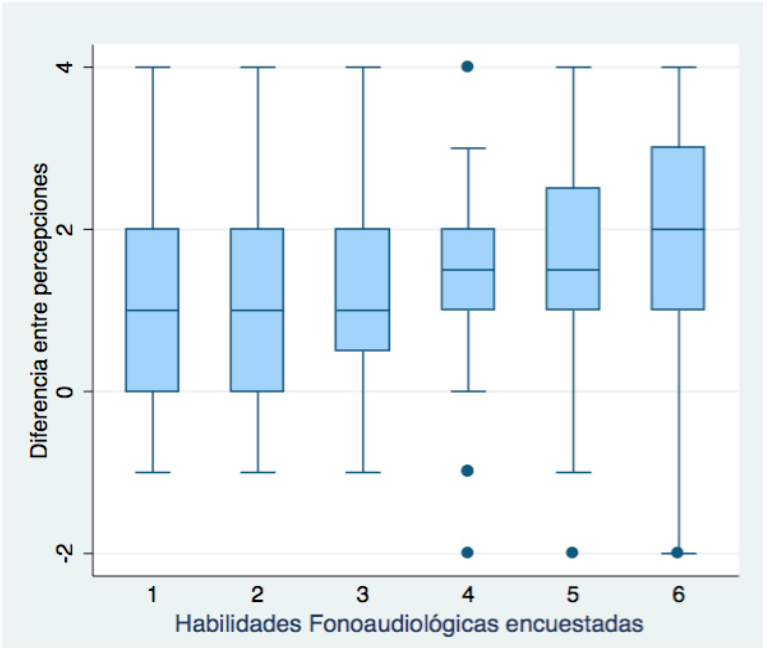

Grafico 3. Diferencias entre las percepciones de desarrollo al egresar y requerimiento laboral en las seis habilidades encuestadas.

\section{Diferencias en las percepciones ajustadas por habilidad y grupo}

Dado que existían diferencias entre las percepciones de algunas habilidades consultadas, se buscó determinar si estas se encontraban influenciadas, además, por el grupo al cual pertenecían los sujetos. Para ello, se creó un modelo estadístico ajustado por tipo de habilidad (seis habilidades) y por grupo. De acuerdo a él, tanto el tipo de habilidad fonoaudiológica $(F=4.45, p<0.001)$ como el grupo al cual pertenecía el encuestado ( $\mathrm{F}=$ 4.43, $\mathrm{p}<0.05)$ resultaron ser predictores significativos de las diferencias encontradas. Los promedios estimados por el modelo (ajustados por tipo de habilidad y grupo) de las diferencias entre ambas percepciones se presentan en el Gráfico 4.

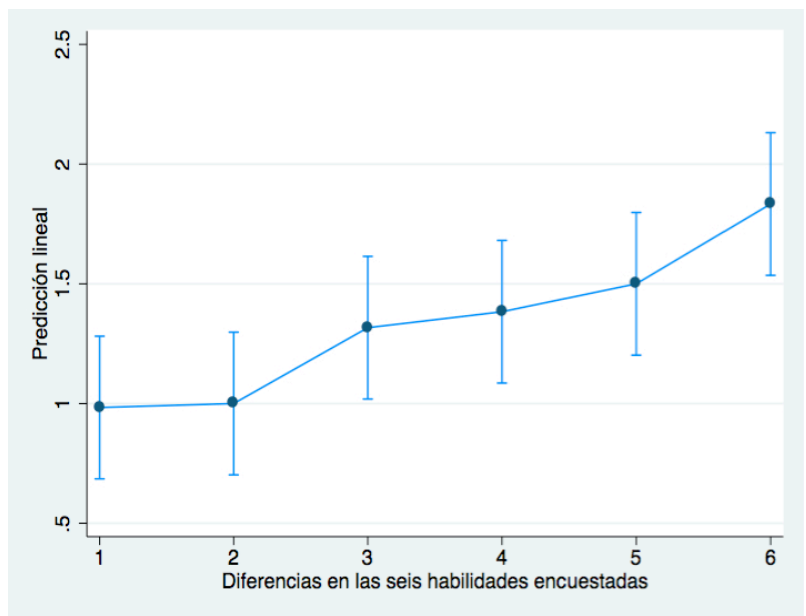

Grafico 4. Promedios marginales con intervalo de confianza del $95 \%$ de las diferencias entre las percepciones (ajustados según habilidad y grupo).

En cuanto a los contrastes entre las diferencias de ambas percepciones, ajustadas según habilidad y tipo de grupo, resultaron significativas las observadas entre las habilidades 5 y 1 ( $F=5.78$, 
$\mathrm{p}<0.05)$ y 6 y $1(\mathrm{~F}=15.64, \mathrm{p}<0.001)$. A su vez, también fue significativo el contraste entre las diferencias entre las dos primeras habilidades y las restantes $(F=$ 8.95, $\mathrm{p}<0.01)$. Estos resultados difieren parcialmente de los datos no ajustados en los que la habilidad 2 presentó diferencias con la 6, en tanto que la habilidad 5 no se diferenciaba de la 1.

Con el objetivo de aclarar en parte los resultados anteriormente señalados, se compararon las diferencias de cada una de las habilidades en relación con el promedio de las diferencias globales. Al respecto, se advirtió que los contrastes significativos se encuentran entre las diferencias de las percepciones de la habilidad $1\left(X^{2}(1)=6.46\right.$, $\mathrm{p}<0.05$ №2 $\left(\mathrm{X}^{2}(1)=5.87, \mathrm{p}<0.05\right)$ y la habilidad 6 $\left(X^{2}(1)=12.84, p<0.001\right)$ y el promedio grupal, como se aprecia en el Gráfico 5.

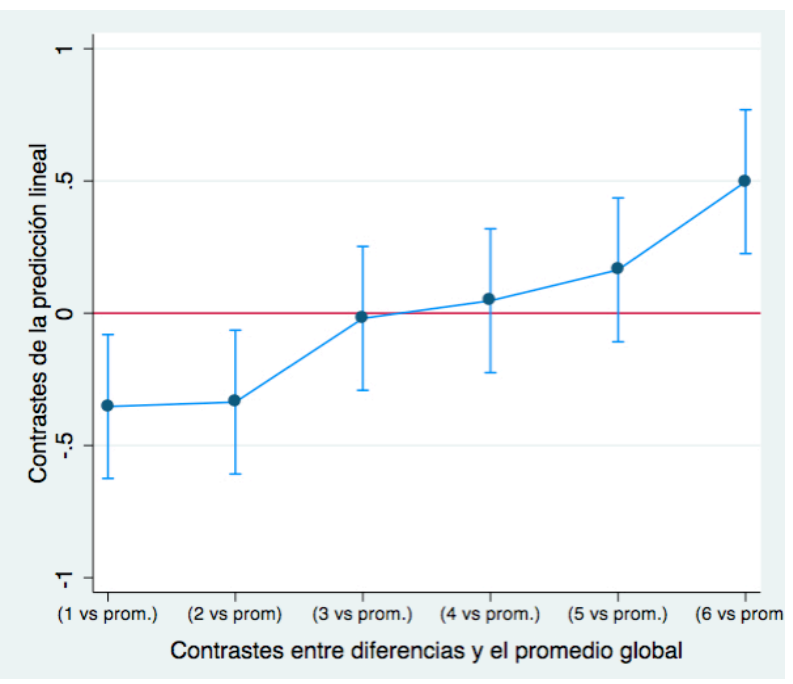

Grafico 5. Contrastes entre las diferencias de las percepciones y el promedio general (línea horizontal), utilizando un intervalo de confianza del $95 \%$.

Como se mencionó previamente, la separación en dos grupos resultó ser un predictor significativo para las diferencias encontradas en cada una de las habilidades $(F=4.43, p<0.05)$. En relación con los promedios estimados, en el grupo №1 fueron significativos las diferencias entre las habilidades 3 y $1(\mathrm{~F}=2.19, \mathrm{p}<0.05) ; 4$ y $1(\mathrm{~F}=2.30, \mathrm{p}<0.05) ; 5$ y $1(\mathrm{~F}=$ $2.74, p<0.01)$ y finalmente 6 y $1(F=3.07, p<0.01)$. En el grupo №2, solo fue significativo el contraste entre las habilidades 6 y $1(\mathrm{~F}=2.52, \mathrm{p}<0.05)$, denotando con ello la homogeneidad existente en este grupo como lo expresa el Gráfico 6.

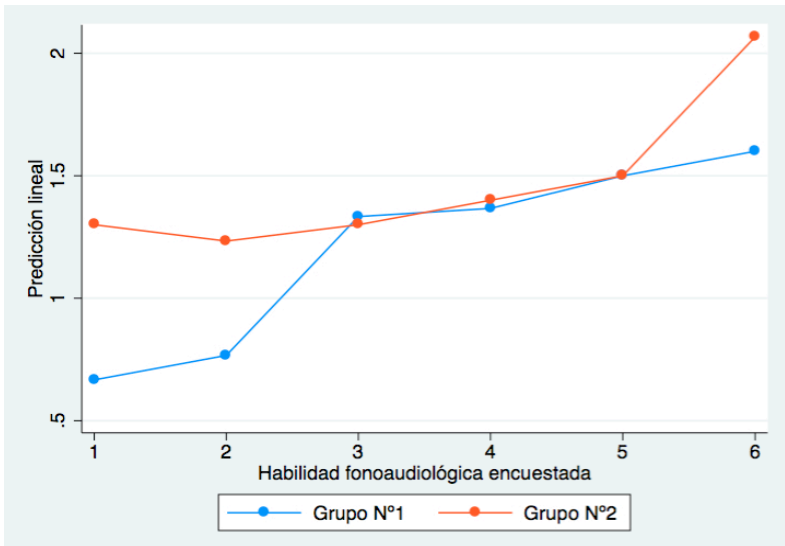

Grafico 6. Promedios estimados de las diferencias entre las percepciones en las seis habilidades y en ambos grupos.

\section{Discusión}

La hipótesis de trabajo propuesta planteaba la existencia de diferencias entre la percepción de la formación universitaria recibida y la percepción acerca de la exigencia por parte del mercado laboral en torno a seis habilidades fonoaudiológicas. A la luz del análisis efectuado, es posible aceptar la anterior premisa, ya que en todas las habilidades consultadas y en los dos grupos constituidos existen diferencias significativas entre ambas percepciones. En efecto, los resultados reflejan una deficiente sintonía entre el mundo universitario y el laboral, la que en parte 
podría explicarse por la utilización de un modelo formativo de carácter tradicional. Dicho modelo, a diferencia del enfoque basado en competencias, dificultaría la adquisición por parte de los estudiantes universitarios de ciertas destrezas profesionales requeridas por el mercado ${ }^{16}$.

Al considerar por separado cada una de las seis habilidades encuestadas y determinar las diferencias entre ambas percepciones, es posible concluir que la habilidad con mayor diferencia fue la 6 (Desarrollar proyectos desde el ámbito fonoaudiológico tanto a nivel público como privado). Mientras que aquellas con menores diferencias fueron las relacionadas directamente con el quehacer profesional del fonoaudiólogo (habilidad 1: "Evaluar el desarrollo comunicativo en las áreas de Voz, Audición, Habla y Lenguaje" y habilidad 2: “Establecer diagnósticos fonoaudiológicos en las áreas de Voz, Audición, Habla y Lenguaje"). Lo anterior también se reflejó al comparar las diferencias entre las percepciones de cada una de las habilidades con respecto al promedio grupal, en donde las habilidades 1 y 2 se encuentran significativamente por debajo del promedio, contrariamente a lo que ocurre con la habilidad 6, la que se ubica significativamente por sobre la media

Una posible explicación a lo anterior se puede encontrar al revisar el perfil profesional de la universidad de procedencia de los encuestados, el cual estuvo vigente hasta el año 2012 y que hasta ese momento no hacía referencia explícita a las habilidades relacionadas con la formulación y desarrollo de proyectos en el ámbito fonoaudiológico.
La diferencia existente entre la percepción de desarrollo al egresar de la universidad y la percepción de requerimiento por parte del mercado laboral mostró estar influenciada por el grupo al cual pertenecía el egresado. Lo anterior podría explicarse por la evolución experimentada en los programas curriculares de la institución formadora, ya que como se mencionó previamente, la carrera de Fonoaudiología de la Universidad de Chile incorporó en el año 1995 un quinto año de estudios y el grado de licenciado en Fonoaudiología ${ }^{12}$. Esto trajo consigo mejoras significativas en los programas académicos, las que podrían verse reflejadas en la disminución de las diferencias entre formación y exigencia laboral que mostraron los egresados después del cambio curricular.

En relación con las implicancias que estos resultados podrían tener es posible señalar que, en primer instancia, permitirían orientar los esfuerzos de las instituciones formadoras hacia las debilidades que perciben sus egresados. Sería recomendable, en este sentido, considerar las falencias que se acaban de señalar al realizar futuras modificaciones curriculares. Además de lo anterior, también se hace necesario discutir acerca de la pertinencia y peso relativo otorgado dentro los perfiles profesionales a aquellas habilidades que no son específicas del quehacer profesional del fonoaudiólogo, pero que son la base de cualquier programa universitario. Las destrezas genéricas o transversales, según estudios realizados a egresados europeos, no son las que preocupan ni por tanto las que se enseñan en los sistemas más tradicionales de educación superior, sin embargo son las que el mercado exige en mayor medida ${ }^{4}$. Así pues, 
los conocimientos específicos constituyen una herramienta útil como punto de partida para los profesionales, aunque los empleadores reconocen cada vez más el valor de las competencias genéricas, tales como las habilidades de comunicación y el trabajo en equipo ${ }^{17}$. En el caso de la Universidad de Chile, podemos afirmar que existió una temprana preocupación por incluir dichas habilidades, lo cual se puede constatar en las declaraciones de perfiles profesionales que incluyen habilidades tales como la capacidad de gestión a nivel personal y laboral, el interés por la investigación científica, así como también evidenciar conocimientos actualizados de la disciplina e integrarse a equipos de trabajo, entre varias otras $^{18}$. Sin embargo, es posible que a pesar de la creciente disposición a mejorar y expandir cada vez más el accionar del profesional, no estén todavía dadas las condiciones de soporte curricular para poder llevar a cabo la formación en otro grupo de habilidades que no sean las propias del quehacer profesional tradicional.

En cuanto a las limitaciones del presente estudio se debe considerar el sesgo de la memoria: algunos encuestados debían recordar eventos acaecidos hacía más de 25 años, lo que perfectamente puede haber distorsionado sus valoraciones. Junto con esto, las percepciones podrían haber sido influenciadas por las exigencias laborales ejercidas a medida que estos profesionales aumentaron su experiencia y desempeñaron funciones gerenciales o ejecutivas (dirección de servicios y/o equipos de trabajo).

Otro factor que debe ser considerado es que las habilidades incluidas en el presente estudio son altamente profesionales, siendo solo la relacionada con la formulación de proyectos (gestión) la que podría ser considerada como genérica o transversal a todo profesional universitario. Es necesario recordar que la versión original del cuestionario propuesto por Cáceres, Didier y Fuentes ${ }^{13}$, se sometió a un análisis exploratorio de factores para la presente investigación. Dicha versión, además de las seis habilidades anteriormente mencionadas, incluyó otras relacionadas en su mayoría con el proceso de gestión e investigación. Estas últimas mostraron una carga factorial pobre y una elevada representación en más de un factor, por lo cual fueron eliminadas. Lo anterior no significa que las habilidades de gestión e investigación carezcan de importancia, sino mas bien que el modo en que se incluyeron en la versión original determinó que no formaran parte del constructo medido. Al incluirlas los resultados no habrían sido válidos ni confiables. Es de esperar que futuros estudios en esta materia aborden una amplia gama de habilidades, tanto genéricas como específicas.

Finalmente, en cuanto a las modificaciones curriculares que aún quedan por realizar, ya lo señalaban tempranamente Maggiolo y Schwalm $\left(1999^{12}\right)$ : «los cambios esperados con la adopción del nuevo enfoque deberían orientar los planes de estudio hacia el logro de habilidades de aprendizaje y de mayor autonomía por parte de los alumnos. En el antiguo modelo, las mallas evolucionaban incorporando nuevos contenidos a medida que el campo laboral se expandía y aumentando notablemente la carga académica de los estudiantes, pero sin producir mejoras significativas en la 
formación de aquellas destrezas relacionadas con el mundo laboral. Por el contrario, en opinión de Biesma y otros $\left(2008^{17}\right)$ los cambios curriculares deberían incluir mejoras en la metodología de la enseñanza, enfatizando precisamente el aprendizaje activo y la integración de habilidades.

\section{Agradecimientos}

El autor de esta publicación agradece a las siguientes personas:

- Fonoaudiólogo Jorge Lizama Godoy, por el apoyo en las diversas correcciones al escrito.

- Fonoaudióloga Claudia Arancibia, por su colaboración desde el inicio de esta investigación.

\section{Referencias}

1. CINDA. (2006). Currículo universitario basado en competencias, Santiago de Chile. Recuperado desde: http://aula.virtual.ucv.cl/aula_virtual/cinda/cdlibros/35

-Currículo Universitario Basado en
Competencias/Currículo Universitario Basado en Competencias.pdf

2. Corvalán, O. y Hawes, G. (2006). Aplicación del enfoque de competencias en la construcción curricular de la Universidad de Talca. Recuperado desde: http://www.rieoei.org/deloslectores/1463Corvalan.pdf

3. García-Aracil, A. \& Van der Velden, R. (2008). Competencies for young European higher education graduates: labor market mismatches and their payoffs. Higher Education, Vol. 55, Issue 2, 219 - 239.

4. Mora, J.G. (2004). La necesidad del cambio educativo para la sociedad del conocimiento. Revista Iberoamericana de Educación, 35, 13-37.

5. Brunner, J.J. y Uribe, D. (2007). Mercados universitarios: el nuevo escenario de la educación superior. Santiago: Universidad Diego Portales.

6. Mora, J.G. (2003). Competencias y empleo de los jóvenes graduados universitarios. Revista de Educación, 330, 157-170.

7. Mora, J.G. (2002). Formación, empleo y demandas laborales: la universidad española en el contexto europeo. En F. Michavila y J. Martínez (eds.), El carácter transversal en la educación universitaria. Cátedra UNESCO de Gestión y Política Universitaria. UPM. Madrid. pp. 151-166.

8. ANECA (2009). Los procesos de inserción laboral de los titulados universitarios en España. Factores de facilitación y obstaculización. Recuperado desde: http://www.aneca.es/media/308144/publi_procesosil. pdf

9. Alonso, L., Rodríguez, C. y Nyssen, J. (2008). El debate sobre las competencias. Una investigación cualitativa en torno a la educación superior y el mercado de trabajo en España. Madrid: Agencia Nacional de Evaluación de la Calidad y Acreditación. 
10. Bell, J. \& Mitchell, R. (2000). Competency-based versus traditional cohort based technical education: a comparison of students' perceptions. Journal of career and technical education, 17, 1, 5-22.

11. Peluffo, M.B. \& Knust, R. (2009). Aproximación a la educación universitaria por competencias en América Latina: ¿Una "fata morgana» o un modelo factible para la realidad latinoamericana? Chile. Recuperado desde: http://mt.educarchile.cl/MT/jjbrunner/archives/2010/0 3/aproximacion_a.html

12. Maggiolo, M. \& Schwalm, E. (1999). Escuela de Fonoaudiología: Notas acerca de su historia. Revista Chilena de Fonoaudiología, 1, 5-10.

13. Comisión Local de Innovación Curricular, Escuela de Fonoaudiología. (2012). Plan de formación Escuela de Fonoaudiología, Chile.

14. Cáceres, I., Didier, B., Fuentes, L. (2010). Relación entre el desarrollo de las habilidades en el ámbito de la gestión y la exigencia del mercado laboral, percibida por los profesionales fonoaudiólogos. Tesis para optar al grado académico de Magíster en Pedagogía Universitaria. Facultad de Educación, Universidad Mayor.
15. Brown, B., Hendrix, S., Hedges, D. and Smith, T. (2012). Multivariate Analysis for the Biobehavioral and Social Sciences: A Graphical Approach. USA: John Wiley \& Sons, Inc.

16. Hu, L-T. and Bentler, P. (1999). Cutoff criteria for fit indexes in covariance structure analysis: Conventional criteria versus new alternatives. Structural Equation Modeling, 6, 1-55.

17. San Martín, V. (2002). La formación en competencias: El desafío de la educación superior en Iberoamérica. Revista Iberoamericana de Educación. Recuperado desde: http://www.rieoei.org/deloslectores/280SanMartin.PD F

18. Biesma, R.G., Pavlova, M., Vaatstra, R., Van Merode, G.G., Czabanowska, K., Smith, T., et al. (2008). Generic versus specific competencies of entry-level public health graduates: Employers perceptions in Poland, the UK and the Netherlands. Advances in Health Sciences Education, 13, 3, 325-343.

19. De Barbieri, Z. (1999). Perfil profesional del Fonoaudiólogo chileno. Revista Chilena de Fonoaudiología, 2, 5-10. 


\section{CUESTIONARIO}

"HABILIDADES FONOAUDIOLÓGICAS Y EXIGENCIA DEL MERCADO LABORAL"

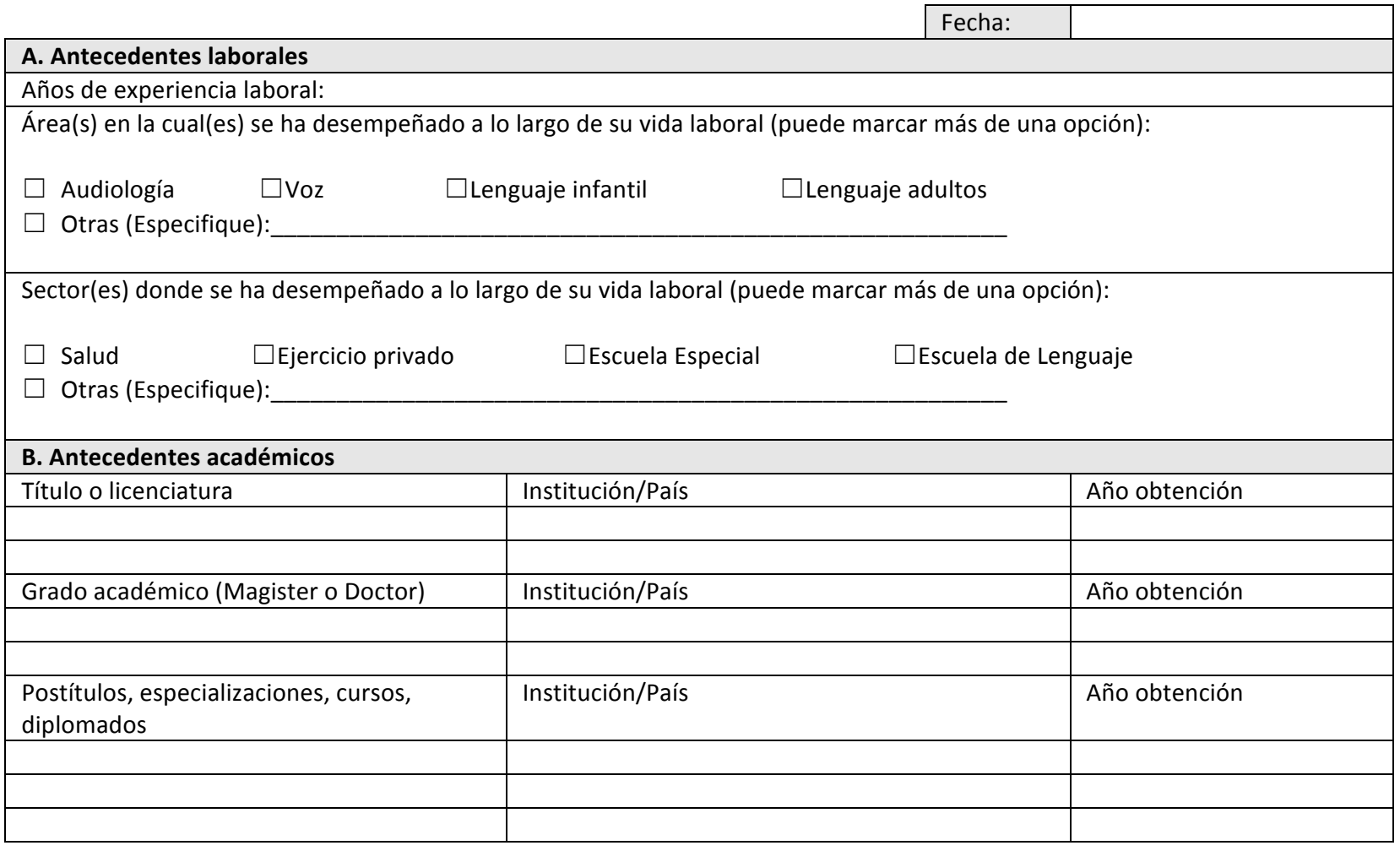

C. Para cada una de las siguientes habilidades fonoaudiológicas; indique el nivel de desarrollo que alcanzaron cuando usted egresó de la universidad (columna izquierda) y el requerimiento o exigencia en el trabajo (columna derecha):

\begin{tabular}{|c|c|c|c|c|c|c|c|c|c|c|}
\hline \multicolumn{5}{|c|}{$\begin{array}{l}\text { Desarrollo } \\
\text { al egreso }\end{array}$} & \multirow[t]{2}{*}{ Habilidades Fonoaudiológicas } & \multicolumn{5}{|c|}{$\begin{array}{l}\text { Requerimiento en el } \\
\text { trabajo }\end{array}$} \\
\hline \multicolumn{2}{|c|}{ Bajo } & \multicolumn{3}{|c|}{ Alto } & & \multicolumn{2}{|c|}{ Bajo } & \multicolumn{3}{|c|}{ Alto } \\
\hline 1 & 2 & 3 & 4 & 5 & $\begin{array}{l}\text { Evaluar el desarrollo comunicativo en las áreas de Voz, Audición, } \\
\text { Habla y Lenguaje. }\end{array}$ & 1 & 2 & 3 & 4 & 5 \\
\hline 1 & 2 & 3 & 4 & 5 & $\begin{array}{l}\text { Establecer diagnósticos fonoaudiológicos en las áreas de Voz, } \\
\text { Audición, Habla y Lenguaje. }\end{array}$ & 1 & 2 & 3 & 4 & 5 \\
\hline 1 & 2 & 3 & 4 & 5 & $\begin{array}{l}\text { Planificar la intervención terapéutica de acuerdo a las necesidades } \\
\text { comunicativas de la persona y su entorno. }\end{array}$ & 1 & 2 & 3 & 4 & 5 \\
\hline 1 & 2 & 3 & 4 & 5 & $\begin{array}{l}\text { Implementar planes de intervención terapéutica de acuerdo a las } \\
\text { necesidades comunicativas de la persona y su entorno. }\end{array}$ & 1 & 2 & 3 & 4 & 5 \\
\hline 1 & 2 & 3 & 4 & 5 & $\begin{array}{l}\text { Evaluar el plan de intervención terapéutica de acuerdo a las } \\
\text { necesidades comunicativas de la persona y su entorno, } \\
\text { readecuándolo cuando sea pertinente. }\end{array}$ & 1 & 2 & 3 & 4 & 5 \\
\hline
\end{tabular}

